\title{
ANH
}

\section{DOAÇÃO DE ÓRGÃOS E TECIDOS PARA TRANSPLANTE: ORGANIZAÇÃO DO SERVIÇO E PARTICIPAÇÃO DO ENFERMEIRO}

\author{
Kelen Mayer Machado', Simone Lysakowski ${ }^{2}$, Rita Catalina Aquino Caregnato ${ }^{3}$, Carine Raquel Blatt 4
}

\section{RESUMO}

Objetivo: o objetivo desse estudo foi relatar a participação dos enfermeiros de uma Organização de Procura de Órgãos (OPO) na unificação do serviço com uma Comissão Intra-Hospitalar de Doação de Órgãos e Tecidos para Transplantes (CIHDOTT).

Método: o relato inclui a experiência de um complexo hospitalar, referência em saúde, da região sul do Brasil, no período de agosto de 2016 a dezembro de 2018, com a reformulação das atividades em três etapas: a) Processos administrativos e assistenciais; b) Atribuições do enfermeiro; e c) Educação permanente.

Resultados: os transplantes de órgãos evoluíram significativamente ao longo dos anos, apresentando um aumento de 2,4\%, na taxa de doadores efetivos por milhão de população (pmp) em 2018. Os enfermeiros das Organização de Procura de Órgãos (OPO) e Comissão Intra-Hospitalar de Doação de Órgãos e Tecidos para Transplantes (CIHDOTT) devem ser capacitados para atuar em cada etapa desse processo, sendo responsáveis por planejar, organizar, coordenar e executar a assistência a esses pacientes. A participação do enfermeiro é fundamental no desenvolvimento das atividades da OPO e CIHDOTT, prestando cuidado aos pacientes e familiares, desenvolvendo atividades de gestão, assistência e educação, tornando-se referência para a equipe de saúde no processo de doação e transplantes.

Conclusão: A atuação desses profissionais na unificação dos dois serviços foi essencial, permitindo melhoria nas rotinas e bons resultados, evidenciados pelo aumento das notificações de possíveis doadores e consequentemente no acréscimo no número de doações possibilitando a melhor organização do serviço e interação com as equipes assistenciais.

Descritores: Transplantes; Cuidados de enfermagem; Obtenção de tecidos e órgãos; Cuidados críticos; Educação continuada.

Key words: Transplants; nursing care; procurement of tissues and organs; critical care; continuing education.

Abstract: Trasplantes; cuidados de enfermería; obtención de tejidos y órganos; atención crítica; educación contínua.

1. Kelen Mayer Machado - Enfermeira. Mestranda em enfermagem pela UFCSPA. Enfermeira na Organização de Procura de Órgãos (OPO) da Santa Casa de Misericórdia de Porto Alegre, Rio Grande de Sul, Brasil. kelenmayer@hotmail.com. ORCID iD: https://orcid.org/0000-0001-9111-2355

2. Simone Lysakowski - Enfermeira. Mestre em Ensino na Saúde pela UFCSPA. Enfermeira na Organização de Procura de Órgãos (OPO) da Santa Casa de Misericórdia de Porto Alegre. Docente na Faculdade Anhanguera Porto Alegre, Rio Grande do Sul, Brasil.silysa@gmail.com. ORCID iD: https://orcid. org/0000-0003-3959-956X

3. Rita Catalina Aquino Caregnato - Enfermeira. Doutora em Educação. Professora do Departamento de Enfermagem da Universidade Federal de Ciências da Saúde de Porto Alegre. Rio Grande de Sul, Brasil. ritac@ufcspa.edu.br. ORCID iD: https://orcid.org/0000-0001-7929-7676

4. Carine Raquel Blatt - Farmacêutica. Doutorado em Farmácia. Professora do Departamento de Farmácia da Universidade Federal de Ciências da Saúde de Porto Alegre. Rio Grande de Sul, Brasil. carine.blatt@gmail.com. ORCID iD: https://orcid.org/0000-0001-5935-1196

\section{O manuscrito se destina a seção de relato de experiência.}

Autora correspondente: Kelen Mayer Machado Av. Independência, 155 - Centro Histórico de Porto Alegre. RS. Brasil. (51)3214-8459. kelenmayer@hotmail.com

Data de submissão: 29/05/2019 Data de aceite: $08 / 07 / 2019$

Como citar esse artigo

MACHADO, K. M. et al. Doação de órgãos e tecidos para transplante: organização do serviço e participação do enfermeiro. Advances in Nursing and Health, v. 1, p. 34-51, Londrina, 2019. 


\section{INTRODUÇÃO}

Os transplantes de órgãos evoluíram significativamente ao longo dos anos, contribuindo como uma terapia cada vez mais efetiva para pacientes portadores de doenças crônicas terminais [1-4] . O sucesso progressivo estimulou $\mathrm{o}$ ingresso de pacientes em lista de espera por um órgão, tornando-se uma das únicas alternativas para doenças anteriormente incuráveis [4-5].

Dados mundiais evidenciam a escassez de órgãos para transplantes [6-8]. A cada ano o número de pessoas em lista de espera é ainda maior que o número de doadores e transplantes realizados [9-11].

No Brasil, no ano de 2018 foi registrado um aumento de $2,4 \%$, na taxa de doadores efetivos por milhão de população (pmp), passando de 16,6 pmp em 2017 para 17,0 pmp em 2018, abaixo da taxa prevista que era de 18,0 doadores pmp. Com isso, fica ainda mais perceptível essa grande desproporção entre o número de pacientes em listas e número de transplante realizados [12]. Em 2018, foram realizados 3.415 transplantes de órgãos enquanto, aproximadamente 33.000 pacientes aguardavam na lista de espera [5].
Com o intuito de ampliar a Política de captação de órgãos e transplantes, bem como aprimorar a busca e identificação dos potenciais doadores e, promover a articulação dos hospitais com as Centrais Estaduais de Transplantes (CET), o Ministério da Saúde (MS) brasileiro, através da Portaria no 1.752 instituiu no ano de 2005, a constituição das Comissões Intra-Hospitalares de Doação de Órgãos e Tecido para Transplantes $(\mathrm{CIH}-$ DOTT) no país, sendo responsabilidade dos hospitais, públicos, privados e filantrópicos, com mais de 80 leitos, ter uma Comissão [13].

Com o objetivo de aumentar o número de doadores, em 2009 o Estado do Rio Grande do Sul (RS) aderiu ao modelo Americano de Organização de Procura de Órgãos (OPO), através da Portaria 2.600, que estabelece a composição de recursos humanos, devendo conter um médico coordenador, enfermeiros e funcionário administrativo, buscando desenvolver um papel supra-hospitalar de atuação em conjunto com as CIHDOTTs de sua abrangência [14-15]. Dessa forma, o RS aderiu a um modelo misto composto por OPO e CIHDOTT, distinguindo-se dos demais estados (Figura 1). 
Figura 1 - Modelo da Organização de Procura de Órgãos (OPO), Comissão Intra-Hospitalar de Doação de Órgãos e Tecidos para Transplantes (CIHDOTT) e misto.

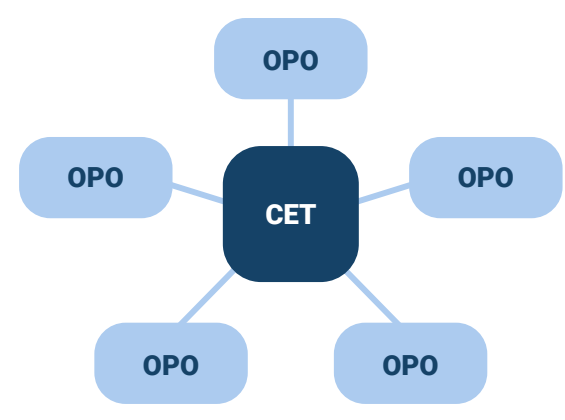

1.a: modelo de OPO

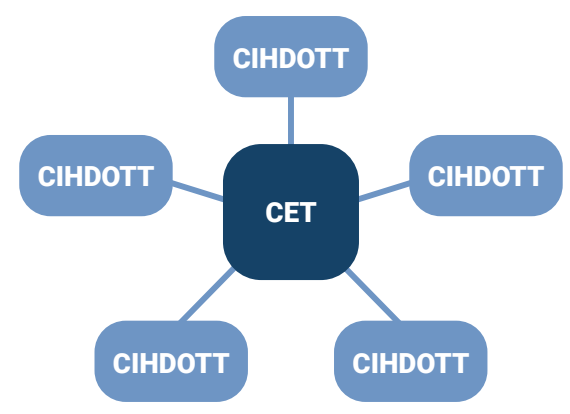

1.b: modelo de CIHDOTT

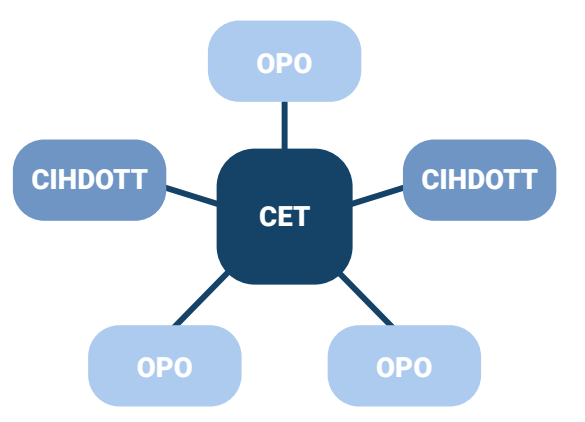

1.c: modelo misto de OPO e CIHDOTT

O processo de doação de órgãos e tecidos pode ser compreendido como um conjunto de etapas que se inicia com a identificação do possível doador e notificação à CET, seguido da efetivação do diagnóstico de morte encefálica [16-17]. Quando não existir contraindicação para doação de órgãos, a entrevista familiar é realizada e, se consentida a doação, seguem-se as demais etapas com a manutenção da viabilidade dos órgãos, avaliação física e do histórico do paciente, investigação laboratorial, e retirada dos órgãos no centro cirúrgico, que culmina com a entrega do corpo aos familiares [1619].

O enfermeiro é apontado como o profissional responsável por planejar, organizar, coordenar e executar a assistência, bem como realizar a supervisão e avaliação dos procedimentos de enfermagem ao doador de 
órgãos, aplicando a Sistematização da Assistência, incluindo o acompanhamento pré, trans e pós-transplante [14,20-22]. Sendo assim, os enfermeiros da OPOs e CIHDOTTs devem ser capacitados para atuar em cada etapa desse processo, possibilitando a promoção da assistência qualificada para esses pacientes, bem como aos cuidadores e familiares, a fim de proporcionar continuidade ao acolhimento fora do ambiente hospitalar $[1,6,23]$.

A atuação das CIHDOTTs mostra-se assertiva na articulação entre os profissionais de uma mesma instituição, o que proporciona melhor interação multiprofissional e conhecimento prévio das rotinas hospitalares, podendo gerar maior segurança para quem executa as atividades. O que pode não acontecer em uma OPO, quando essa não está inserida nas instituições de saúde de abrangência.

Considerando a importância da assistência de enfermagem em todas as etapas do transplante e da necessidade de organização das ações relacionadas ao processo de doação, relatar a vivência de enfermeiros no processo de unificação de uma OPO e CIHDOTT, torna-se significativo para compreensão referente a essa temática.
Diante deste contexto, o presente estudo tem como objetivo relatar a participação do enfermeiro de uma OPO na reestruturação e unificação com uma CIHDOTT.

\section{MÉTODO}

Trata-se de um relato de experiência, ocorrido em um complexo hospitalar de caráter filantrópico, referência em saúde na Região Sul do Brasil, que possui cerca de 1.300 leitos, sendo 90 de Unidades de Tratamento Intensivo (UTIs) e 116 de emergência, distribuídos em sete hospitais direcionados às especialidades de clínica médica, cirurgia geral, pediatria, pneumologia, neurologia, cardiologia, oncologia e transplantes.

As atividades da CIHDOTT nesse hospital iniciaram-se no ano de 1999, antes mesmo da obrigatoriedade vigente na legislação, composta por uma equipe multiprofissional, onde estavam inseridos um médico, três assistentes social, um enfermeiro e um funcionário administrativo, passando por mudanças no quadro funcional com o passar dos anos. Em 2011, instituiu-se uma OPO no mesmo complexo hospitalar, que tinha como atribuição o acompanhamento de 22 hospitais externos, conduzida por uma equipe 
composta por duas enfermeiras, um funcionário administrativo e um médico coordenador, conforme determinado pela legislação, através da Portaria 2.600/2009 [15].

No segundo semestre de 2016, ambos setores passaram por reestruturação solicitada pela instituição que previa contenção de despesas por meio da redução no quadro funcional, com a proposta de unir os serviços de CIHDOTT e OPO. Desta forma, a equipe sofreu alterações na sua composição e no ano de 2017 passou a atuar com dois médicos (que já atuavam, um como coordenador da OPO e outro da CIHDOTT), dois enfermeiros exclusivos do serviço, dois enfermeiros que atuam em UTI e que auxiliam nos plantões noturnos e final de semana e um funcionário administrativo.

Essa equipe passou a exercer as atividades relacionadas à captação, doação e transplante nos sete hospitais do complexo e nos 22 hospitais externos.

\section{RESULTADO}

Com a unificação da CIHDOTT e OPO houve a organização das atividades de forma intra e extra hospitalar, atendendo ao que fora re- gulamentado diante das Portarias que determinam cada serviço.

Dessa maneira, apresenta-se o relato da experiência que ocorreu no período de agosto de 2016 a dezembro 2018, em três etapas denominadas: a) Processos administrativos e assistenciais; b) Atribuições do enfermeiro na CIHDOTT e OPO e c) Educação permanente.

\section{PROCESSOS ADMINISTRATIVOS E ASSISTENCIAIS}

No âmbito intra-hospitalar, os profissionais que exercem as atividades como $\mathrm{CIH}^{-}$ DOTT conforme a Portaria no 1.752 de 2005 [13] , têm as seguintes atribuições 1) detectar possíveis doadores de órgãos e tecidos no hospital onde está inserido; 2) viabilizar o diagnóstico de morte encefálica, conforme a Resolução no 2.173 de 2017do Conselho Federal de Medicina [24]; 3) criar e aprimorar rotinas para oferecer aos familiares de pacientes falecidos no hospital, a possibilidade da doação de córneas e outros tecidos até seis horas após a PCR; 4) articular-se com a Central Estadual de Transplante (CET), do estado respectivo para organizar o processo de doação e captação de órgãos e tecidos; 5) responsabilizar-se pela educação continuada dos funcionários da instituição sobre os as- 
pectos de doação e transplantes de órgãos e tecidos; 6) articular-se com todas as unidades que possuam recursos importantes para atender aos casos de possível doação; e 7) capacitar em conjunto com a CET e Sistema Nacional de Transplantes (SNT), os funcionários do estabelecimento hospitalar para atuarem no processo de doação, bem como acolher as famílias enlutadas e realizar entrevista familiar de solicitação e doação de órgãos e tecidos.

Estabeleceu-se um cronograma de reuniões semanais, participando os médicos coordenadores e os enfermeiros da CIHDOTT/ OPO. As reuniões foram realizadas a fim de abordar assuntos referentes ao: protocolo de morte encefálica (ME), estabelecimento de novas metas para a notificação e doação de órgãos, planejamento das atividades de competência da equipe, atualização dos Procedimentos Operacionais Padrão (POPs) para busca ativa de doadores e entrevista familiar para doação de órgãos, seguindo assim o modelo padronizado pela instituição que fora desenvolvido juntamente com o setor de qualidade, com o objetivo de sistematizar e informatizar os processos.

Uma das estratégias para aumentar o número de notificações de ME foi conhecer o perfil dos pacientes atendidos nos hospitais de abrangência da OP e CIHDOTT, através da implementação de busca ativa. Assim, a equipe passou a realizar visitas nas UTIs e emergências quatro vezes por semana, em turnos alternados, com o objetivo de identificar pacientes com critérios para realização do protocolo de ME.

Outra importante estratégia para aumentar o número de notificações foi a implementação da busca ativa intra hospitalar pelo enfermeiro no sistema informatizado do hospital. Diariamente, é acessado o prontuário eletrônico de todos os pacientes em ventilação mecânica, internados nas UTIs e emergências, monitorando e observando a evolução do quadro clínico, principalmente avaliação da escala de coma Glasgow.

$\mathrm{Na}$ instituição onde discorre esse relato, foram notificados à CET (Gráfico 1): 
Gráfico 1 - Comparação do número de notificações realizadas e doações efetivadas na Organização de Procura de Órgãos (OPO) e na Comissão Intra-Hospitalar de Doação de Órgãos e Tecidos para Transplantes (CIHDOTT).

\section{NOTIFICAÇÕES X DOAÇÕES CIHDOTT}

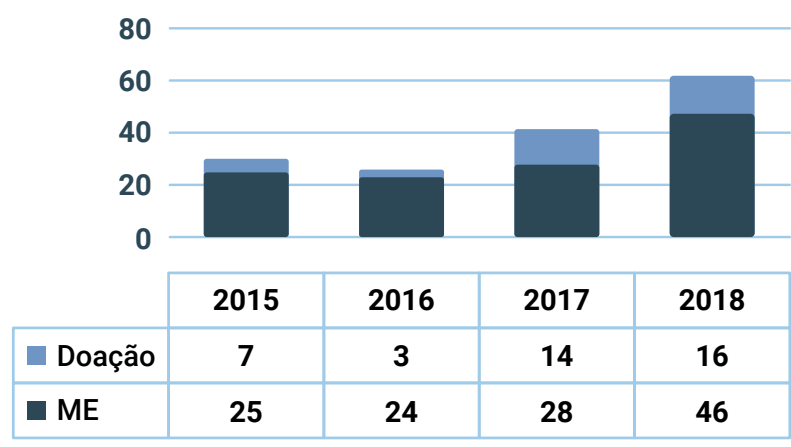

Gráfico 1a: Notificações e doações CIHDOTT

Em gráfico 1a e 1b as notificações de morte encefálica correspondem ao determinado na legislação, preconizando um mínimo de $30 \%$ de efetivação da doação de órgãos sobre o total de casos notificados à CET [13].

Quanto aos desfechos dos protocolos de ME realizados pela CIHDOTT no ano de 2015 a 2018, é possível verificar um aumento no número de notificações no ano de 2018, nunca atingido anteriormente, consequentemente a doação também aumentou, porém não na mesma proporção das noti-

\section{NOTIFICAÇÕES X DOAÇÃO OPO}

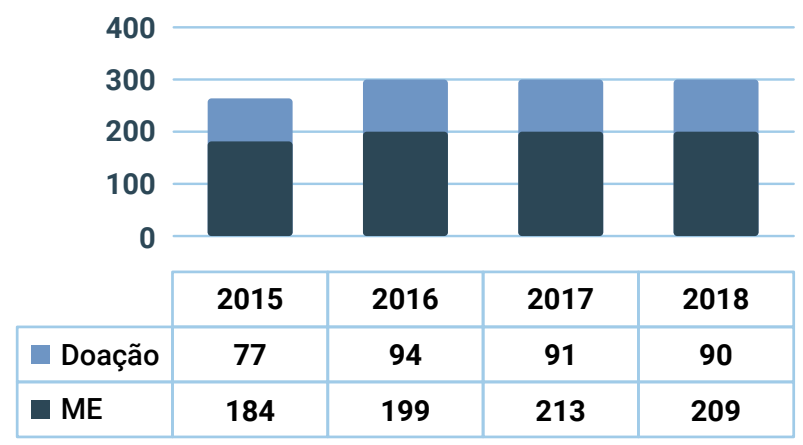

Gráfico 1b: Notificações e doações OPO

ficações, o que pode estar relacionado ao alto número de contraindicação médica, que totalizaram 18 casos no último ano. A instituição é referência em atendimento especializado no Estado, recebendo pacientes com as mais diversas patologias que muitas vezes são contraindicações para doação de órgãos como tumores, sorologia positiva para HIV (vírus da imunodeficiência viral), infecções não controladas, doenças raras entre outros (Gráfico 2). 
Gráfico 2 - Desfechos dos protocolos de Morte Encefálica da Comissão Intra-Hospitalar de Doação de Órgãos e Tecidos para Transplantes (CIHDOTT).

\section{DESFECHOS DOS PROTOCOLOS DE ME DA CIHDOTT}

\begin{tabular}{|l|c|c|c|}
\hline \multicolumn{1}{c|}{} & & & \\
\hline
\end{tabular}

Quando considerados os números da CIHDOTT, entre os anos de 2015 e 2018, a permanência de notificações de ME e doação é vista como positiva, diante da queda dos números nacionais (Gráfico 3).

Gráfico 3 - Desfechos dos protocolos de Morte Encefálica daOrganização de Procura de Órgãos (OPO).

\section{DESFECHOS DOS PROTOCOLOS DE ME DA OPO}

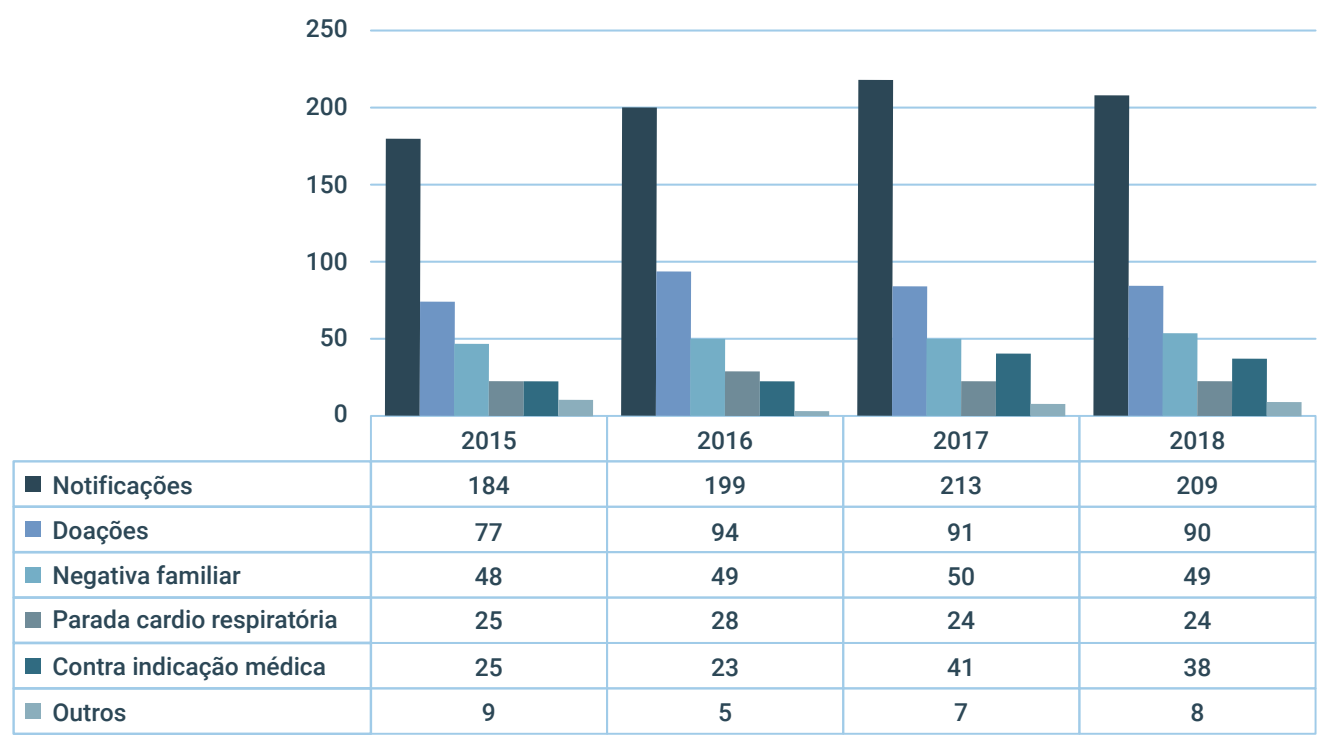


Importante mencionar que a notificação da ME é compulsória, devendo ser realizada mesmo nos casos de contraindicação médica, de acordo com a Resolução no

2.173, de 23 de novembro de 2017.

\section{ATRIBUIÇÕES DO ENFERMEIRO DA OPO E CIHDOTT}

O enfermeiro é referência para a equipe de saúde e pacientes, atuando no processo

de doação e transplantes de forma integrada com a equipe multiprofissional. Para organizar a assistência do enfermeiro durante o processo de doação de órgãos e tecidos, sumarizaram-se as atividades exercidas, conforme apresentadas no quadro 1 .

\section{Quadro 1 - Atividades do enfermeiro no acompanhamento do Protocolo de Morte Encefálica e Doação de Órgãos.}

\section{NOTIFICAÇÃO DE MORTE ENCEFÁLICA}

- Receber contato do profissional da UTI ou da Emergência onde se encontra o potencial doador informando o caso.

- Ir até o hospital levando todos os formulários necessários.

- Apresentar-se ao médico e enfermeira de plantão.

- Verificar no prontuário: causa do coma, possível contraindicação para abertura do protocolo de ME, hipotermia, hipotensão, ou uso de drogas depressoras do sistema nervoso central.

- Solicitar ao médico da unidade que informe à família da gravidade do caso e possibilidade de evolução para ME. 


\section{DIAGNÓSTICO DE MORTE ENCEFÁLICA}

- Acompanhar a realização do primeiro exame clínico e preenchimento do termo de ME.

- Notificar à CET informando sobre histórico de saúde do paciente e encaminhar a documentação.

- Solicitar ao médico plantonista que comunique à família sobre o resultado do exame clínico.

- Organizar a logística para realização do exame complementar de imagem.

- Acompanhar a realização do exame de imagem, conferindo o preenchimento correto do laudo, nome do paciente, data e horário de realização.

- Certificar-se de que o médico conversou com familiares informando o óbito.

- Apoiar e auxiliar a família enlutada quanto ao entendimento da morte e exames realizados para o diagnóstico de ME.

\section{ENTREVISTA FAMILIAR PARA DOAÇÃO DE ÓRGÃOS}

- Realizar a entrevista familiar para a doação de órgãos e tecidos, no momento em que estes estiverem em condições.

- Preencher o Termo de Autorização para Doação de Órgãos e Tecidos para aqueles que concordarem com a doação, solicitar a assinatura dos familiares, conferindo com o documento de identidade. Encaminhar cópia dos documentos do potencial doador de órgãos à CET, que deve validar a documentação e dar seguimento.

- Orientar a família quanto às próximas etapas do processo, auxiliando com documentação legal, atestado de óbito ou encaminhamento ao IML.

- Informar o número do telefone do plantão da CIHDOTT para qualquer dúvida ou esclarecimento. 


\section{ENTREVISTA FAMILIAR PARA DOAÇÃO DE ÓRGÃOS}

- Disponibilizar-se à família em caso de dúvidas quanto ao processo de doação, combinando com a mesma a comunicação ao término.

- Coletar e encaminhar exames ao laboratório e monitorar os resultados para encaminhar à CET.

- Auxiliar, orientar e supervisionar a manutenção do doador até o momento da captação.Realizar a entrevista familiar para a doação de órgãos e tecidos, no momento em que estes estiverem em condições.

\section{CAPTAÇÃO DE ÓRGÃOS E TECIDOS}

- Organizar juntamente com a CET o horário para início da captação dos órgãos e agendar a sala do centro cirúrgico.

- Conferir todos os documentos originais que comprovam a morte encefálica e a autorização familiar para doação de órgãos.

- Acompanhar o transporte do doador até o centro cirúrgico, juntamente com o médico e técnico de enfermagem, atentando para a manutenção e viabilidade dos órgãos.

- Auxiliar a família na liberação do corpo após o término da captação dos órgãos.

- Informar à CET do término da captação de órgãos. 


\section{ACOMPANHAMENTO PÓS-DOAÇÃO DE ÓRGÃOS E TECIDOS}

- Informar a família sobre os órgãos e tecidos doados.

- Acompanhar os resultados de exames posteriores à captação e informar à CET.

- Realizar evolução de todo o processo e arquivar na sala da CIHDOTT juntamente com a cópia do protocolo de morte encefálica e exames laboratoriais.

- Solicitar a CET documento informando sobre os órgãos e tecidos que foram utilizados ou descartados e anexar junto ao processo.

O acompanhamento do enfermeiro nesse processo favorece a possibilidade de efetivação da doação, garantindo tanto o direito do diagnóstico de ME ao paciente quanto o direito de decisão das famílias dos falecidos. O enfermeiro deve proporcionar o acolhimento familiar independente da doação de órgãos $[1,3]$.

\section{EDUCAÇÃo PERMANENTE}

Conforme determinado pela Portaria no 2.600 de 2009 [14], as equipes que atuam em prol da doação de órgão para Transplantes, sendo elas OPOs e CIHDOTTs, são responsáveis pela educação permanente das equipes assistenciais e de apoio, promovendo a educação continuada de todos os profis- sionais para a compreensão do processo de doação de órgãos e tecidos.

Devido à importância da educação permanente, no ano de 2017 realizaram-se diferentes atividades de ensino aos profissionais da saúde inseridos nas instituições de saúde de abrangência. Iniciadas com capacitações direcionadas para enfermeiros das Unidades de Tratamento Intensivo (UTI), Emergências e médicos residentes da neurologia e medicina intensiva. As capacitações aconteceram no centro de simulação realística da instituição, sendo possível simular o atendimento em uma UTI ou emergência, equipado com manequins simuladores que possibilitaram a realização dos testes de morte encefálica, bem como sala multimídia para a apresen- 
tação teórica. Além das realizadas na instituição, os enfermeiros também organizaram e promoveram cursos de capacitação extra hospitalares, atendendo as necessidades dos hospitais de abrangência.

As capacitações tiveram duração de duas horas entre teoria e prática, contemplando o diagnóstico de ME e a importância dos profissionais neste processo. Organizaram-se as turmas com no máximo 10 participantes para melhor interação da equipe. No total foram capacitados 90 enfermeiros e 40 residentes de medicina.

Em novembro de 2017, com a nova resolução que determina a realização do diagnóstico de $M E$, algumas alterações importantes foram pontuadas, tais como o tempo para realização do diagnóstico de ME e habilitação dos médicos para realização do diagnóstico (24) .

Desta forma foi necessária a realização de novas capacitações para habilitar os médicos a realizarem o diagnóstico, sendo o enfermeiro responsável pela organização das atividades em conjunto com os médicos coordenadores da OPO e CIHDOTT.

As capacitações, conforme estabelecido pelo Conselho Federal de Medicina (CFM) devem totalizar 8 horas, dividias em 4 horas teóricas e 4 horas práticas. Aproximadamente 200 médicos foram capacitados até o final de 2018, sendo possível acreditar que desta forma o número de notificações no mesmo ano, tenha aumentado devido a quantidade de médicos capacitados para realização do diagnóstico de $M E$, o que pode ter proporcionado um maior envolvimento das equipes assistenciais após as capacitações.

Dando sequência às atividades educativas, essa comissão participou juntamente com o marketing da instituição, do lançamento da campanha "Vida - o Show tem que continuar", que teve como objetivo incentivar e estimular a população a pensar sobre a doação de órgãos para transplantes. Confeccionaram-se camisetas e botons, além da distribuição de materiais educativo, contendo informações e esclarecendo a comunidade sobre o tema. A campanha ocorreu nas dependências da instituição, atingindo emissoras de rádio e televisão em nível nacional, além de outdoors distribuídos pela cidade.

Essa comissão também promoveu palestras em escolas de ensino médio e fundamental da região, além de aulas em universidades parceiras, sendo possível a divulgação do assunto e esclarecimento dos estudantes 
sobre a sua importância para a sociedade como multiplicador de informações corretas. Também foram realizadas atividades durante seminários e eventos realizados por outras instituições, com o intuito de levar ainda mais informações aos interessados pelo assunto.

\section{DISCUSSÃO}

Nesse estudo foi possível relatar a importante experiência de enfermeiros, na unificação de uma OPO e CIHDOTT, descrevendo as etapas de reformulação dos processos administrativos e assistenciais, atuação na educação permanente, bem como distinguir o papel do enfermeiro na CIHDOTT e OPO [13-14].

A padronização dos processos permite orientar as ações entre os profissionais de forma segura, promovendo padrões de cuidados de enfermagem para a assistência no processo de doação e transplante de órgãos e tecidos [3].

Como indicadores para o monitoramento das ações da equipe definiram-se: número de óbitos em UTI e emergência, número de notificações de $M E$, número de contraindicações, número de entrevistas realizadas, número de negativas familiares e número de doações. Esses são indicadores utilizados mundialmente por serviços que trabalham com doação de órgãos, sendo importante a monitorização mensal pela equipe de enfermagem $[4,7]$.

Torna-se positiva essa junção, uma vez que é possível designar atividades semelhantes, porém em âmbitos diferente, á mesmos profissionais, tornando-os mais experiente diante dos processos, uma vez que participam com maior frequência das etapas diagnósticas e de doação de órgãos.

Acredita-se que o trabalho desenvolvido, após a unificação dos dois serviços fora relevante e distinto, refletindo nos resultados anuais, uma vez que as notificações e doações de órgãos no último ano aumentaram em relação aos anos anteriores.

A unificação dos serviços permite aos profissionais da enfermagem e médico, discussão construtiva de ações e tomada de decisão diante doas situações de dificuldades encontradas no processo de doação e transplantes, proporcionando pensamento crítico e desenvolvimento profissional e pessoal [17-18].

Os enfermeiros elaboram mensalmente um relatório para as áreas de apoio, com os 
números de transplantes realizados na instituição, direcionado ao laboratório, imunologia, banco de sangue, UTI, unidade de internação de transplantes, gerências e direção geral, bem como para a CET, que realiza os levantamentos estatísticos do estado [13].

Os enfermeiros atuam na linha de frente com as equipes assistenciais, pacientes e familiares, divulgando, orientando e incentivando a doação de órgãos nas instituições de saúde que atendem, empenhando-se ativamente em conjunto com as UTIs e emergências para a identificação de possíveis doadores de órgãos. As capacitações dos profissionais médicos e de enfermagem que atuam nas UTIs e Emergências foi fundamental para o aumento no número de notificações e maior envolvimento das equipes neste processo de doação $[1,18]$.

A educação permanente é uma eficiente ferramenta que possibilita melhorias no processo de trabalho, permitindo a ampliação e atualização dos conhecimentos, favorecendo o diálogo e a troca de experiência de forma participativa entre profissionais e a população [25].

\section{CONCLUSÃO}

A equipe segue buscando novas estratégias para incentivar e estimular as equipes assistenciais em relação à doação de órgãos, uma vez que são elas que iniciam o processo de notificação para a OPO e CIHDOTT, sendo fundamentais também na manutenção do possível doador.

A unificação desses dois serviços permitiu a importante troca de saberes dos profissionais envolvidos, que ao longo das discussões de casos oportunizou melhor preparo e segurança para situações semelhantes na posteridade.

O enfermeiro desempenha importante papel prestando cuidado aos pacientes e familiares atendidos por ele, desenvolvendo atividades de gestão, assistência e educação, respeitando os preceitos éticos e legais, tornando-se referência para a equipe de saúde no processo de doação e transplantes 


\section{REFERÊNCIAS}

1. Moreira WC, Barbosa TMA, Ribeiro WRA, Damasceno CKCS, Alencar DC, Vieira SKSF. Assistência de enfermagem no processo de doação de órgãos e transplantes. Rev Prev Infecç Saúde. 2016;2(1-2):32-42. doi: 10.26694/repis.v2i1-2.4381.

2. Westphal GA, Garcia VD, Souza RL, Franke $C A$, Vieira KD, Birckholz VR, et al. Diretrizes para manutenção de múltiplos órgãos no potencial doador adulto falecido. Rev Bras Ter Intensiva. 2016;28(3):220-255. doi: 10.5935/0103-507X.20160049.

3. Zúñiga-Fajuri Alejandra. Transparência e transplantes: é possível? Dilemas bioéticos da alocação de órgãos. Bioeth Act [Internet]. 2017 jul [citado 2019 set 03]; 23 (2): 237- 243. Disponível em:https://scielo.conicyt.cl/scielo. php?script $=$ sci_arttext\&amp; pid $=$ S1726569X2017000200237\&amp;Ing=en. http:// dx. doi.org/10.4067/S1726 $569 \times 2017000200237$.

4. Scientific Registry of Transplant Recipients. SRTR/OPTN annual data report: statistics on donation and transplantation in the United Status [Internet]. Departament of Health and Human Services (US); 2018 [citado 2019 mar 20]. Disponível em: https://www.srtr.org/ reports-tools/srtroptn-annual-data-report/.

5. Associação Brasileira de Transplantes de Órgãos (ABTO). Dimensionamento dos Transplantes no Brasil e em cada estado no período de 2011 a 2018. RBT [Internet]. 2018 [citado 2019 mar 20]; 24(4):1-89. Disponível em: http://www.abto.org.br/abtov03/Upload/ file/RBT/2018/Lv_RBT-2018.pdf
6. Tech AW, Cruz LV, Cornelli M, do Vale Pereira C, Bertoglia J, Brasil B, et al. Implementing activities developer by the organ transplantation Academic Society of the Hospital Dom Vicente Scherer: a pilot study. Transplant Proc. 2016Set;48(7):2253-7. doi: 10.1016/j.transproceed.2016.06.009

7. Organ Procurement and Transplantation Network (OPTN). Board of Directors Meeting. Annual data report. Departament of health and human services (US); 2018. [citado 2019 Mar 20]. Disponível em: https://optn. transplant.hrsa.gov/

8. Hodgson R, Young AL, Attia MA, Lodge JPA. Impact of a National Controlled Donation After Circulatory Death (DCD) Program on Organ Donation in the United Kingdom: A 10 Year Study. Am J Transplant . 2017 dez; 17 (12): 3172-3182. doi: 10.1111 / ajt.14374.

9. Murakami M, Fukuma S, Ikezoe M, Nakamura M, Yamazaki S, et al. Effect of an educational program on attitudes towards deceased organ donation. Ann Transplant. 2015 may;20:26978. doi: 10.12659/АOT.893325

10. Fiorelli $A L$, Stolf $N A$, Pego-Fernandes $P M$, Oliveira Júnior JL, Santos RH, Contreras CA, et al. Recommendations for use of marginal donors in heart transplantation: Brazilian Association of Organs Transplantation guideline. Transplant Proc. 2011jan-feb;43(1):211-5. doi: 10.1016/j.transproceed.2010.12.047.

11. Silva FAA, Cunha DSP, Lira JAC, Ribeiro JF, Campelo GVS, Nunes BMVT. Morte encefálica e manutenção de órgãos: conhecimento dos profissionais intensivistas. Rev Enferm UFPE. 2018 jan;12(1):518. doi: 10.5205/1981-8963-v12i01a2513 0p51-58-2018. 
12. Associação Brasileira de Transplantes de Órgãos (ABTO). Dimensionamento dos Transplantes no Brasil e em cada estado (2009-2016). RBT [Internet]. 2016 [citado 2019 abr 15]; 22(4):1-89. Disponível em: http://www.abto.org.br/abtov03/Upload/file/ RBT/2016/RBT2016-leitura.pdf.

13. Ministerio da Saúde (BR). Portaria no 1.752, de 23 de setembro de 2005. Determina a constituição de Comissão Intra-Hospitalar de Doação de Órgãos e Tecidos para Transplante em todos os hospitais públicos, privados e filantrópicos com mais de 80 leitos [Internet]. Brasília, DF: Ministério da Saúde; 2005 [citado 2019 mar 20]. Disponível em: http://bvsms. saude.gov.br/bvs/saudelegis/gm/2005/ prt1752_23_09_2005.html.

14. Ministerio da Saúde (BR). Portaria no 2.600, de 21 de outubro de 2009. Aprova o Regulamento Técnico do Sistema Nacional de Transplantes [Internet]. Brasília, DF: Ministério da Saúde; 2009 [citado 2019 mar 20]. Disponível em: http://bvsms.saude.gov.br/bvs/saudelegis/ gm/2009/prt2600_21_10_2009.html.

15. Ministerio da Saúde (BR). Portaria no 2.601, de 21 de outubro de 2009. Institui, no âmbito do Sistema Nacional de Transplantes, o Plano Nacional de Implantação de Organizações de Procura de Órgãos e Tecidos - OPO [Internet]. Brasília, DF: Ministério da Saúde; 2009 [citado 2019 mar 20]. Disponível em: http://bvsms. saude.gov.br/bvs/saudelegis/gm/2009/ prt2601_21_10_2009.html.

16. Fonseca PIMN, Tavares CMM. O preparo emocional dos profissionais de saúde na entrevista familiar: estudo hermenêutico. Online Braz J Nurs. 2014 set;13(4):496-506. doi $10.17665 / 1676-4285.2014 v 13 n 4$

17. Morais TR, Morais MR. Doação de órgãos: é preciso educar para avançar. Saúde Debate. 2012out-dez;36(95):633-9.

doi: $10.1590 /$ S0103-11042012000400015

18. Bianchi M, Accinellia LG, Silva MA, Menegócio AM. Identificação dos diagnósticos de enfermagem ao paciente potencial doador de órgãos. Uniciências. 2015 dez;19(2):174-80. doi: $10.17921 / 1415-5141.2015 v 19 n 2 p \% 25 p$.

19. Araújo C, Santos JAV, RAP Rodrigues, Guidi Júnior LRG. O papel do profissional de enfermagem na doação de órgãos. Rev Saúde Foco [Internet]. 2017 [citado 2019 jan 10]; 9:533-51. Disponível em: http:// portal.unisepe.com.br/unifia/wp-content/ uploads/sites/10001/2018/06/061_papel_ profissional_enfermagem.pdf.

20. Conselho Federal de Enfermagem (BR). Resolução COFEN no 292/2004. Normatiza a atuação do enfermeiro na captação e transplante de órgãos e tecidos [Internet]. Rio de Janeiro: COFEN; 2004 [citado 2018 mar 10]. Disponível em: http://www.cofen.gov.br/ resoluo-cofen-2922004_4328.html.

21. Garcia CD, Garcia VD, Pereira JD. Manual de doação e transplantes: informações práticas sobre todas as etapas do processo de doação de órgãos e transplante. Porto Alegre: Libretos; 2017.

22. Halldorson J, Roberts JP. Decadal analysis of deceased organ donation in Spain and the United States linking an increased donation rate and the utilization of older donors. Liver Transpl. 2013 sep;19(9):981-6. doi: 10.1002/ It. 23684

23. Andrade JDA de, Brito AC de, Lira GG, Fernandes FECV, Melo RA. Experiences and strategies of an organ procurement organization vivências e estratégias de uma organização de procura de órgãos. Rev Enferm UFPE on line. 
2018abr;12(4):857-64. doi: 10.5205/19818963-v12i4a110257p857-864-2018.

24. Conselho Federal de Medicina (BR). Resolução no 2.173, de 23 de novembro de 2017. Define os critérios do diagnóstico de morte encefálica [Internet]. Diário Oficial da União, Brasilia; 2017. Seção 1, p. 50-275. [citado 2019 mar 18]. Disponível em: https://saude.rs.gov.br/ upload/arquivos/carga20171205/19140504resolucao-do-conselho-federal-demedicina-2173-2017.pdf

25. Silva EPB, Nunes FS, Bueno JLS, Silva MG, Silva PRB, Santos RC, Santos SSS. Educação permanente como instrumento de trabalho do profissional de saúde. Rev Remecs [Internet]. 2017 [citado 2019 mar 15]; 2(2):41-6. Disponível em: http://www.revistaremecs. com.br/index.php/remecs/article/view/10/ pdf_1. 